\title{
Finding Common Solutions of a Variational Inequality, a General System of Variational Inequalities, and a Fixed-Point Problem via a Hybrid Extragradient Method
}

\author{
Lu-Chuan Ceng, ${ }^{1}$ Sy-Ming Guu, ${ }^{2}$ and Jen-Chih Yao ${ }^{3}$ \\ ${ }^{1}$ Department of Mathematics, Shanghai Normal University, Scientific Computing Key Laboratory of \\ Shanghai Universities, Shanghai 200234, China \\ ${ }^{2}$ Department of Business Administration, College of Management, Yuan-Ze University, Taoyuan Hsien, \\ Chung-Li City 330, Taiwan \\ ${ }^{3}$ Department of Applied Mathematics, National Sun Yat-Sen University, Kaohsiung 804, Taiwan
}

Correspondence should be addressed to Sy-Ming Guu, iesmguu@saturn.yzu.edu.tw

Received 25 September 2010; Accepted 20 December 2010

Academic Editor: Jong Kim

Copyright (C) 2011 Lu-Chuan Ceng et al. This is an open access article distributed under the Creative Commons Attribution License, which permits unrestricted use, distribution, and reproduction in any medium, provided the original work is properly cited.

We propose a hybrid extragradient method for finding a common element of the solution set of a variational inequality problem, the solution set of a general system of variational inequalities, and the fixed-point set of a strictly pseudocontractive mapping in a real Hilbert space. Our hybrid method is based on the well-known extragradient method, viscosity approximation method, and Mann-type iteration method. By constrasting with other methods, our hybrid approach drops the requirement of boundedness for the domain in which various mappings are defined. Furthermore, under mild conditions imposed on the parameters we show that our algorithm generates iterates which converge strongly to a common element of these three problems.

\section{Introduction}

Let $H$ be a real Hilbert space with inner product $\langle\cdot, \cdot\rangle$ and norm $\|\cdot\|$. Let $C$ be a nonempty closed convex subset of $H$ and $S: C \rightarrow C$ be a self-mapping on $C$. We denote by $\operatorname{Fix}(S)$ the set of fixed points of $S$ and by $P_{C}$ the metric projection of $H$ onto $C$. Moreover, we also denote by $\mathbf{R}$ the set of all real numbers. For a given nonlinear operator $A: C \rightarrow H$, we consider the following variational inequality problem of finding $x^{*} \in C$ such that

$$
\left\langle A x^{*}, x-x^{*}\right\rangle \geq 0, \quad \forall x \in C
$$


The solution set of the variational inequality (1.1) is denoted by $\operatorname{VI}(A, C)$. Variational inequality theory has been studied quite extensively and has emerged as an important tool in the study of a wide class of obstacle, unilateral, free, moving, equilibrium problems. See, for example, [1-21] and the references therein.

For finding an element of $\operatorname{Fix}(S) \cap \operatorname{VI}(A, C)$ when $C$ is closed and convex, $S$ is nonexpansive and $A$ is $\alpha$-inverse strongly monotone, Takahashi and Toyoda [22] introduced the following Mann-type iterative algorithm:

$$
x_{n+1}=\alpha_{n} x_{n}+\left(1-\alpha_{n}\right) S P_{C}\left(x_{n}-\lambda_{n} A x_{n}\right), \quad \forall n \geq 0,
$$

where $P_{C}$ is the metric projection of $H$ onto $C, x_{0}=x \in C,\left\{\alpha_{n}\right\}$ is a sequence in $(0,1)$, and $\left\{\lambda_{n}\right\}$ is a sequence in $(0,2 \alpha)$. They showed that, if $\operatorname{Fix}(S) \cap \operatorname{VI}(A, C) \neq \emptyset$, then the sequence $\left\{x_{n}\right\}$ converges weakly to some $z \in \operatorname{Fix}(S) \cap \operatorname{VI}(A, C)$. Nadezhkina and Takahashi [23] and Zeng and Yao [24] proposed extragradient methods motivated by Korpelevič [25] for finding a common element of the fixed point set of a nonexpansive mapping and the solution set of a variational inequality problem. Further, these iterative methods were extended in [26] to develop a new iterative method for finding elements in $\operatorname{Fix}(S) \cap \operatorname{VI}(A, C)$.

Let $B_{1}, B_{2}: C \rightarrow H$ be two mappings. Now we consider the following problem of finding $\left(x^{*}, y^{*}\right) \in C \times C$ such that

$$
\begin{array}{ll}
\left\langle\mu_{1} B_{1} y^{*}+x^{*}-y^{*}, x-x^{*}\right\rangle \geq 0, & \forall x \in C, \\
\left\langle\mu_{2} B_{2} x^{*}+y^{*}-x^{*}, x-y^{*}\right\rangle \geq 0, & \forall x \in C,
\end{array}
$$

which is called a general system of variational inequalities where $\mu_{1}>0$ and $\mu_{2}>0$ are two constants. The set of solutions of problem (1.3) is denoted by GSVI $\left(B_{1}, B_{2}, C\right)$. In particular, if $B_{1}=B_{2}=A$, then problem (1.3) reduces to the problem of finding $\left(x^{*}, y^{*}\right) \in C \times C$ such that

$$
\begin{array}{ll}
\left\langle\mu_{1} A y^{*}+x^{*}-y^{*}, x-x^{*}\right\rangle \geq 0, & \forall x \in C, \\
\left\langle\mu_{2} A x^{*}+y^{*}-x^{*}, x-y^{*}\right\rangle \geq 0, & \forall x \in C,
\end{array}
$$

which was defined by Verma [27] (see also [28]) and is called the new system of variational inequalities. Further, if $x^{*}=y^{*}$ additionally, then problem (1.4) reduces to the classical variational inequality problem (1.1).

Ceng et al. [29] studied the problem (1.3) by transforming it into a fixed-point problem. Precisely and for easy reference, we state their results in the following lemma and theorem.

Lemma CWY (see [29]). For given $\bar{x}, \bar{y} \in C,(\bar{x}, \bar{y})$ is a solution of problem (1.3) if and only if $\bar{x}$ is a fixed point of the mapping $G: C \rightarrow C$ defined by

$$
G(x)=P_{C}\left[P_{C}\left(x-\mu_{2} B_{2} x\right)-\mu_{1} B_{1} P_{C}\left(x-\mu_{2} B_{2} x\right)\right], \quad \forall x \in C,
$$

where $\bar{y}=P_{C}\left(\bar{x}-\mu_{2} B_{2} \bar{x}\right)$. In particular, if the mapping $B_{i}: C \rightarrow H$ is $\mu_{i}$-inverse strongly monotone for $i=1,2$, then the mapping $G$ is nonexpansive provided $\mu_{i} \in\left(0,2 \mu_{i}\right)$ for $i=1,2$. 
Throughout this paper, the fixed-point set of the mapping $G$ is denoted by $\Gamma$. Utilizing Lemma CWY, they introduced and studied a relaxed extragradient method for solving problem (1.3).

Theorem CWY (see [29, Theorem 3.1]). Let C be a nonempty closed convex subset of a real Hilbert space $H$. Let the mapping $B_{i}: C \rightarrow H$ be $\beta_{i}$-inverse strongly monotone for $i=1,2$. Let $S: C \rightarrow C$ be a nonexpansive mapping with $\operatorname{Fix}(S) \cap \Gamma \neq \emptyset$. Suppose $x_{1}=u \in C$ and $\left\{x_{n}\right\}$ is generated by

$$
\begin{gathered}
y_{n}=P_{C}\left(x_{n}-\mu_{2} B_{2} x_{n}\right), \\
x_{n+1}=\alpha_{n} u+\beta_{n} x_{n}+\gamma_{n} S P_{C}\left(y_{n}-\mu_{1} B_{1} y_{n}\right),
\end{gathered}
$$

where $\mu_{i} \in\left(0,2 \beta_{i}\right)$ for $i=1,2$, and $\left\{\alpha_{n}\right\},\left\{\beta_{n}\right\},\left\{\gamma_{n}\right\}$ are three sequences in $[0,1]$ such that

(i) $\alpha_{n}+\beta_{n}+\gamma_{n}=1$, for all $n \geq 1$;

(ii) $\lim _{n \rightarrow \infty} \alpha_{n}=0, \sum_{n=0}^{\infty} \alpha_{n}=\infty$;

(iii) $0<\liminf _{n \rightarrow \infty} \beta_{n} \leq \lim \sup _{n \rightarrow \infty} \beta_{n}<1$.

Then $\left\{x_{n}\right\}$ converges strongly to $\bar{x}=P_{\mathrm{Fix}(S) \cap \Gamma} u$ and $(\bar{x}, \bar{y})$ is a solution of problem (1.3), where $\bar{y}=P_{C}\left(\bar{x}-\mu_{2} B_{2} \bar{x}\right)$. literature.

It is clear that the above result unifies and extends some corresponding results in the

Based on the relaxed extragradient method and viscosity approximation method, Yao et al. [30] proposed and analyzed an iterative algorithm for finding a common element of the solution set of the general system (1.3) of variational inequalities and the fixed-point set of a strictly pseudocontractive mapping in a real Hilbert space $H$.

Theorem YLK (see [30, Theorem 3.2]). Let $C$ be a nonempty bounded closed convex subset of a real Hilbert space $H$. Let the mapping $B_{i}: C \rightarrow H$ be $\mu_{i}$-inverse strongly monotone for $i=1,2$. Let $S: C \rightarrow C$ be a $k$-strictly pseudocontractive mapping such that $\operatorname{Fix}(S) \cap \Gamma \neq \emptyset$. Let $Q: C \rightarrow C$ be a $\rho$-contraction with $\rho \in[0,1 / 2)$. For given $x_{0} \in C$ arbitrarily, let the sequences $\left\{x_{n}\right\},\left\{y_{n}\right\}$, and $\left\{z_{n}\right\}$ be generated iteratively by

$$
\begin{gathered}
z_{n}=P_{C}\left(x_{n}-\mu_{2} B_{2} x_{n}\right), \\
y_{n}=\alpha_{n} Q x_{n}+\left(1-\alpha_{n}\right) P_{C}\left(z_{n}-\mu_{1} B_{1} z_{n}\right), \\
x_{n+1}=\beta_{n} x_{n}+\gamma_{n} P_{C}\left(z_{n}-\mu_{1} B_{1} z_{n}\right)+\delta_{n} S y_{n}, \quad \forall n \geq 0,
\end{gathered}
$$

where $\mu_{i} \in\left(0,2 \beta_{i}\right)$ for $i=1,2$ and $\left\{\alpha_{n}\right\},\left\{\beta_{n}\right\},\left\{\gamma_{n}\right\},\left\{\delta_{n}\right\}$ are four sequences in $[0,1]$ such that

$$
\begin{aligned}
& \text { (i) } \beta_{n}+\gamma_{n}+\delta_{n}=1 \text { and }\left(\gamma_{n}+\delta_{n}\right) k \leq \gamma_{n}<(1-2 \rho) \delta_{n} \text { for all } n \geq 0 \text {; } \\
& \text { (ii) } \lim _{n \rightarrow \infty} \alpha_{n}=0 \text { and } \sum_{n=0}^{\infty} \alpha_{n}=\infty \text {; } \\
& \text { (iii) } 0<\liminf _{n \rightarrow \infty} \beta_{n} \leq \lim \sup _{n \rightarrow \infty} \beta_{n}<1 \text { and } \liminf _{n \rightarrow \infty} \delta_{n}>0 \text {; } \\
& \text { (iv) } \lim _{n \rightarrow \infty}\left(\gamma_{n+1} /\left(1-\beta_{n+1}\right)-\gamma_{n} /\left(1-\beta_{n}\right)\right)=0 \text {. }
\end{aligned}
$$

Then the sequence $\left\{x_{n}\right\}$ generated by (1.7) converges strongly to $\bar{x}=P_{\mathrm{Fix}(S) \cap \Gamma} \cdot Q \bar{x}$ and $(\bar{x}, \bar{y})$ is a solution of the general system (1.3) of variational inequalities, where $\bar{y}=P_{C}\left(\bar{x}-\mu_{2} B_{2} \bar{x}\right)$. 
Motivated by the above work, in this paper, we introduce an iterative algorithm for finding a common element of the solution set of the variational inequality (1.1), the solution set of the general system (1.3) and the fixed-point set of the strictly pseudocontractive mapping $S: C \rightarrow C$ via a hybrid extragradient method based on the well-known extragradient method, viscosity approximation method, and Mann-type iteration method, that is,

$$
\begin{gathered}
z_{n}=P_{C}\left(x_{n}-\lambda_{n} A x_{n}\right), \\
y_{n}=\alpha_{n} Q x_{n}+\left(1-\alpha_{n}\right) P_{C}\left[P_{C}\left(z_{n}-\mu_{2} B_{2} z_{n}\right)-\mu_{1} B_{1} P_{C}\left(z_{n}-\mu_{2} B_{2} z_{n}\right)\right], \\
x_{n+1}=\beta_{n} x_{n}+\gamma_{n} y_{n}+\delta_{n} S y_{n}, \quad \forall n \geq 0,
\end{gathered}
$$

where $\left\{\lambda_{n}\right\} \subset(0, \infty),\left\{\alpha_{n}\right\},\left\{\beta_{n}\right\},\left\{\gamma_{n}\right\},\left\{\delta_{n}\right\} \subset[0,1]$ such that $\beta_{n}+\gamma_{n}+\delta_{n}=1$ for all $n \geq 0$. Moreover, we prove that the studied iterative algorithm converges strongly to an element of $\operatorname{Fix}(S) \cap \Gamma \cap \operatorname{VI}(A, C)$ under some mild conditions imposed on algorithm parameters. Our method improves and extends Yao et al. [30, Theorem 3.2] in the following aspects:

(i) the problem of finding an element of $\operatorname{Fix}(S) \cap \Gamma$ in [30, Theorem 3.2] is extended to the the problem of finding an element of $\operatorname{Fix}(S) \cap \Gamma \cap \mathrm{VI}(A, C)$;

(ii) the requirement of boundedness of $C$ in [30, Theorem 3.2] is removed;

(iii) the condition $\left(\gamma_{n}+\delta_{n}\right) k \leq \gamma_{n}<(1-2 \rho) \delta_{n}$, for all $n \geq 0$ in [30, Theorem 3.2] is replaced by the one $\left(\gamma_{n}+\delta_{n}\right) k \leq \gamma_{n}$, for all $n \geq 0$;

(iv) the argument of Step 5 in the proof of [30, Theorem 3.2] is simplified under the lack of the condition $\gamma_{n}<(1-2 \rho) \delta_{n}$, for all $n \geq 0$;

(v) our iterative algorithm is similar to but different from the one of [30, Theorem 3.2] because the problem of finding an element of $\operatorname{Fix}(S) \cap \Gamma \cap \operatorname{VI}(A, C)$ is more challenging than the problem of finding an element of $\operatorname{Fix}(S) \cap \Gamma$ in [30, Theorem 3.2].

\section{Preliminaries}

In this section, we collect some notations and lemmas. Let $C$ be a nonempty closed convex subset of a real Hilbert space $H$. A mapping $A: C \rightarrow H$ is called monotone if

$$
\langle A x-A y, x-y\rangle \geq 0, \quad \forall x, y \in C .
$$

A mapping $A: C \rightarrow H$ is called Lipschitz continuous if there exists a real number $L>0$ such that

$$
\|A x-A y\| \leq L\|x-y\|, \quad \forall x, y \in C .
$$

Recall that a mapping $A: C \rightarrow H$ is called $\alpha$-inverse strongly monotone if there exists a real number $\alpha>0$ such that

$$
\langle A x-A y, x-y\rangle \geq \alpha\|A x-A y\|^{2}, \quad \forall x, y \in C .
$$


It is clear that every inverse strongly monotone mapping is a monotone and Lipschitz continuous mapping. Also, recall that a mapping $S: C \rightarrow C$ is said to be $k$-strictly pseudocontractive if there exists a constant $0 \leq k<1$ such that

$$
\|S x-S y\|^{2} \leq\|x-y\|^{2}+k\|(I-S) x-(I-S) y\|^{2}, \quad \forall x, y \in C
$$

For such a case, we also say that $S$ is a $k$-strict pseudo-contraction [31]. It is clear that, in a real Hilbert space $H$, inequality (2.4) is equivalent to the following:

$$
\langle S x-S y, x-y\rangle \leq\|x-y\|^{2}-\frac{1-k}{2}\|(I-S) x-(I-S) y\|^{2}, \quad \forall x, y \in C
$$

This immediately implies that if $S$ is a $k$-strictly pseudocontractive mapping, then $I-S$ is $(1-k) / 2$-inverse strongly monotone; see [32] for more details. We use Fix $(S)$ to denote the set of fixed points of $S$. It is well known that the class of strict pseudo-contractions strictly includes the class of nonexpansive mappings which are mappings $S: C \rightarrow C$ such that $\|S x-S y\| \leq\|x-y\|$, for all $x, y \in C$. A mapping $Q: C \rightarrow C$ is called a contraction if there exists a constant $\rho \in[0,1)$ such that $\|Q x-Q y\| \leq \rho\|x-y\|$ for all $x, y \in C$. that

For every point $x \in H$, there exists a unique nearest point in $C$, denoted by $P_{C} x$ such

$$
\left\|x-P_{C} x\right\| \leq\|x-y\|, \quad \forall y \in C
$$

The mapping $P_{C}$ is called the metric projection of $H$ onto $C$. It is well known that $P_{C}$ is a nonexpansive mapping and satisfies

$$
\left\langle x-y, P_{C} x-P_{C} y\right\rangle \geq\left\|P_{C} x-P_{C} y\right\|^{2}, \quad \forall x, y \in H .
$$

It is known that $P_{C} x$ is characterized by the following property:

$$
\left\langle x-P_{C} x, y-P_{C} x\right\rangle \leq 0, \quad \forall x \in H, y \in C .
$$

In order to prove the main result in this paper, we will need the following lemmas in the sequel.

Lemma 2.1 (see [33]). Let $\left\{x_{n}\right\}$ and $\left\{y_{n}\right\}$ be bounded sequences in a Banach space $X$ and let $\left\{\beta_{n}\right\}$ be a sequence in $[0,1]$ with $0<\liminf _{n \rightarrow \infty} \beta_{n} \leq \lim \sup _{n \rightarrow \infty} \beta_{n}<1$. Suppose $x_{n+1}=\left(1-\beta_{n}\right) y_{n}+\beta_{n} x_{n}$ for all integers $n \geq 0$ and $\lim \sup _{n \rightarrow \infty}\left(\left\|y_{n+1}-y_{n}\right\|-\left\|x_{n+1}-x_{n}\right\|\right) \leq 0$. Then, $\lim _{n \rightarrow \infty}\left\|y_{n}-x_{n}\right\|=0$.

Lemma 2.2 (see [34, Proposition 2.1]). Let $C$ be a nonempty closed convex subset of a real Hilbert space $H$ and $S: C \rightarrow C$ be a self-mapping of $C$.

(i) If $S$ is a $k$-strict pseudocontractive mapping, then $S$ satisfies the Lipschitz condition

$$
\|S x-S y\| \leq \frac{1+k}{1-k}\|x-y\|, \quad \forall x, y \in C .
$$


(ii) If $S$ is a $k$-strict pseudocontractive mapping, then the mapping $I-S$ is demiclosed at 0 , that is, if $\left\{x_{n}\right\}$ is a sequence in $C$ such that $x_{n} \rightarrow \tilde{x}$ weakly and $(I-S) x_{n} \rightarrow 0$ strongly, then $(I-S) \tilde{x}=0$.

(iii) If $S$ is $k$-(quasi-)strict pseudo-contraction, then the fixed-point set $\operatorname{Fix}(S)$ of $S$ is closed and convex so that the projection $P_{\mathrm{Fix}(S)}$ is well defined.

Lemma 2.3 (see [9, Lemma 2.1]). Let $\left\{s_{n}\right\}$ be a sequence of nonnegative real numbers satisfying the condition

$$
s_{n+1} \leq\left(1-\alpha_{n}\right) s_{n}+\alpha_{n} \beta_{n}, \quad \forall n \geq 0
$$

where $\left\{\alpha_{n}\right\},\left\{\beta_{n}\right\}$ are sequences of real numbers such that

(i) $\left\{\alpha_{n}\right\} \subset[0,1]$ and $\sum_{n=0}^{\infty} \alpha_{n}=\infty$, or equivalently,

$$
\prod_{n=0}^{\infty}\left(1-\alpha_{n}\right):=\lim _{n \rightarrow \infty} \prod_{k=1}^{n}\left(1-\alpha_{k}\right)=0
$$

(ii) $\lim \sup _{n \rightarrow \infty} \beta_{n} \leq 0$; or

(ii) $\sum_{n=0}^{\infty} \alpha_{n} \beta_{n}$ is convergent.

Then, $\lim _{n \rightarrow \infty} s_{n}=0$.

Lemma 2.4 (see [30]). Let $C$ be a nonempty closed convex subset of a real Hilbert space $H$. Let $S: C \rightarrow C$ be a $k$-strictly pseudocontractive mapping. Let $\gamma$ and $\delta$ be two nonnegative real numbers. Assume $(\gamma+\delta) k \leq \gamma$. Then

$$
\|\gamma(x-y)+\delta(S x-S y)\| \leq(\gamma+\delta)\|x-y\|, \quad \forall x, y \in C .
$$

The following lemma is an immediate consequence of an inner product.

Lemma 2.5. In a real Hilbert space $H$, there holds the inequality

$$
\|x+y\|^{2} \leq\|x\|^{2}+2\langle y, x+y\rangle, \quad \forall x, y \in H
$$

Let $A$ be a monotone mapping of $C$ into $H$. In the context of the variational inequality problem the characterization of projection (2.8) implies that

$$
u \in V I(A, C) \Longleftrightarrow u=P_{C}(u-\lambda A u), \quad \forall \lambda>0 .
$$

It is also known that a set-valued mapping $T: H \rightarrow 2^{H}$ is called monotone iffor all $x, y \in H, f \in T x$ and $g \in T y$ imply that $\langle x-y, f-g\rangle \geq 0$. A monotone set-valued mapping $T: H \rightarrow 2^{H}$ is maximal if its graph $\mathrm{Gph}(T)$ is not properly contained in the graph of any other monotone set-valued mapping. It is known that a monotone set-valued mapping $T: H \rightarrow 2^{H}$ is maximal if and only if for $(x, f) \in H \times H,\langle x-y, f-g\rangle \geq 0$ for every $(y, g) \in G p h(T)$ implies that $f \in T x$. Let $A$ be a 
monotone and Lipschitz continuous mapping of $C$ into $H$. Let $N_{C} v$ be the normal cone to $C$ at $v \in C$, that is,

$$
N_{C} v=\{w \in H:\langle v-u, w\rangle \geq 0, \forall \in C\} .
$$

Define

$$
T v= \begin{cases}A v+N_{C} v & \text { if } v \in C, \\ \emptyset & \text { if } v \notin C .\end{cases}
$$

It is known that in this case the mapping $T$ is maximal monotone, and $0 \in T v$ if and only if $v \in$ $\operatorname{VI}(A, C)$; see [35] for more details.

\section{Main Results}

The main idea for showing strong convergence of the sequence $\left\{x_{n}\right\}$ generated by (1.8) to an element of $\operatorname{VI}(A, C)$ is first to transform the variational inequality problem (1.1) into the zero point problem of a maximal monotone mapping $T$ and then to derive the strong convergence of $\left\{x_{n}\right\}$ to a zero of $T$ by using the technique in [10]. We are now in a position to state and prove the main result in this paper.

Theorem 3.1. Let $C$ be a nonempty closed convex subset of a real Hilbert space $H$. Let $A: C \rightarrow H$ be $\alpha$-inverse strongly monotone and $B_{i}: C \rightarrow H$ be $\beta_{i}$-inverse strongly monotone for $i=1,2$. Let $S: C \rightarrow C$ be a k-strictly pseudocontractive mapping such that $\operatorname{Fix}(S) \cap \Gamma \cap V I(A, C) \neq \emptyset$. Let $Q: C \rightarrow C$ be a $\rho$-contraction with $\rho \in[0,1 / 2)$. For given $x_{0} \in C$ arbitrarily, let the sequences $\left\{x_{n}\right\},\left\{y_{n}\right\}$ and $\left\{z_{n}\right\}$ be generated iteratively by

$$
\begin{gathered}
z_{n}=P_{C}\left(x_{n}-\lambda_{n} A x_{n}\right), \\
y_{n}=\alpha_{n} Q x_{n}+\left(1-\alpha_{n}\right) P_{C}\left[P_{C}\left(z_{n}-\mu_{2} B_{2} z_{n}\right)-\mu_{1} B_{1} P_{C}\left(z_{n}-\mu_{2} B_{2} z_{n}\right)\right], \\
x_{n+1}=\beta_{n} x_{n}+\gamma_{n} y_{n}+\delta_{n} S y_{n}, \quad \forall n \geq 0,
\end{gathered}
$$

where $\mu_{i} \in\left(0,2 \beta_{i}\right)$ for $i=1,2,\left\{\lambda_{n}\right\} \subset(0,2 \alpha]$ and $\left\{\alpha_{n}\right\},\left\{\beta_{n}\right\},\left\{\gamma_{n}\right\},\left\{\delta_{n}\right\} \subset[0,1]$ such that

(i) $\beta_{n}+\gamma_{n}+\delta_{n}=1$ and $\left(\gamma_{n}+\delta_{n}\right) k \leq \gamma_{n}$ for all $n \geq 0$;

(ii) $\lim _{n \rightarrow \infty} \alpha_{n}=0$ and $\sum_{n=0}^{\infty} \alpha_{n}=\infty$;

(iii) $0<\liminf _{n \rightarrow \infty} \beta_{n} \leq \lim \sup _{n \rightarrow \infty} \beta_{n}<1$ and $\liminf _{n \rightarrow \infty} \delta_{n}>0$;

(iv) $\lim _{n \rightarrow \infty}\left(\gamma_{n+1} /\left(1-\beta_{n+1}\right)-\gamma_{n} /\left(1-\beta_{n}\right)\right)=0$;

(v) $0<\liminf _{n \rightarrow \infty} \lambda_{n} \leq \limsup _{n \rightarrow \infty} \lambda_{n}<2 \alpha$ and $\lim _{n \rightarrow \infty}\left|\lambda_{n+1}-\lambda_{n}\right|=0$.

Then the sequence $\left\{x_{n}\right\}$ generated by (3.1) converges strongly to $\bar{x}=P_{\mathrm{Fix}(S) \cap \Gamma \cap V I(A, C)} \cdot Q \bar{x}$ and $(\bar{x}, \bar{y})$ is a solution of the general system (1.3) of variational inequalities, where $\bar{y}=P_{C}\left(\bar{x}-\mu_{2} B_{2} \bar{x}\right)$.

Proof. We divide the proof into several steps.

Step 1. $\left\{x_{n}\right\}$ is bounded. 

and

Indeed, take $x^{*} \in \operatorname{Fix}(S) \cap \Gamma \cap \operatorname{VI}(A, C)$ arbitrarily. Then $S x^{*}=x^{*}, x^{*}=P_{C}\left(x^{*}-\lambda_{n} A x^{*}\right)$

$$
x^{*}=P_{C}\left[P_{C}\left(x^{*}-\mu_{2} B_{2} x^{*}\right)-\mu_{1} B_{1} P_{C}\left(x^{*}-\mu_{2} B_{2} x^{*}\right)\right] .
$$

Since $A: C \rightarrow H$ be $\alpha$-inverse strongly monotone and $0<\lambda_{n} \leq 2 \alpha$, we have for all $n \geq 0$,

$$
\begin{aligned}
\left\|z_{n}-x^{*}\right\|^{2} & =\left\|P_{C}\left(x_{n}-\lambda_{n} A x_{n}\right)-P_{C}\left(x^{*}-\lambda_{n} A x^{*}\right)\right\|^{2} \\
& \leq\left\|\left(x_{n}-\lambda_{n} A x_{n}\right)-\left(x^{*}-\lambda_{n} A x^{*}\right)\right\|^{2} \\
& =\left\|\left(x_{n}-x^{*}\right)-\lambda_{n}\left(A x_{n}-A x^{*}\right)\right\|^{2} \\
& \leq\left\|x_{n}-x^{*}\right\|^{2}-\lambda_{n}\left(2 \alpha-\lambda_{n}\right)\left\|A x_{n}-A x^{*}\right\|^{2} \\
& \leq\left\|x_{n}-x^{*}\right\|^{2} .
\end{aligned}
$$

For simplicity, we write $y^{*}=P_{C}\left(x^{*}-\mu_{2} B_{2} x^{*}\right)$ and $u_{n}=P_{C}\left(z_{n}-\mu_{2} B_{2} z_{n}\right)$ for all $n \geq 0$. Since $B_{i}: C \rightarrow H$ be $\beta_{i}$-inverse strongly monotone for $i=1,2$ and $0<\mu_{i}<2 \beta_{i}$ for $i=1$, 2, we know that for all $n \geq 0$,

$$
\begin{aligned}
\| P_{C}[ & \left.P_{C}\left(z_{n}-\mu_{2} B_{2} z_{n}\right)-\mu_{1} B_{1} P_{C}\left(z_{n}-\mu_{2} B_{2} z_{n}\right)\right]-x^{*} \|^{2} \\
= & \| P_{C}\left[P_{C}\left(z_{n}-\mu_{2} B_{2} z_{n}\right)-\mu_{1} B_{1} P_{C}\left(z_{n}-\mu_{2} B_{2} z_{n}\right)\right] \\
& \quad-P_{C}\left[P_{C}\left(x^{*}-\mu_{2} B_{2} x^{*}\right)-\mu_{1} B_{1} P_{C}\left(x^{*}-\mu_{2} B_{2} x^{*}\right)\right] \|^{2} \\
\leq & \|\left[P_{C}\left(z_{n}-\mu_{2} B_{2} z_{n}\right)-\mu_{1} B_{1} P_{C}\left(z_{n}-\mu_{2} B_{2} z_{n}\right)\right] \\
& \quad-\left[P_{C}\left(x^{*}-\mu_{2} B_{2} x^{*}\right)-\mu_{1} B_{1} P_{C}\left(x^{*}-\mu_{2} B_{2} x^{*}\right)\right] \|^{2} \\
= & \|\left[P_{C}\left(z_{n}-\mu_{2} B_{2} z_{n}\right)-P_{C}\left(x^{*}-\mu_{2} B_{2} x^{*}\right)\right] \\
& \quad-\mu_{1}\left[B_{1} P_{C}\left(z_{n}-\mu_{2} B_{2} z_{n}\right)-B_{1} P_{C}\left(x^{*}-\mu_{2} B_{2} x^{*}\right)\right] \|^{2} \\
\leq & \left\|P_{C}\left(z_{n}-\mu_{2} B_{2} z_{n}\right)-P_{C}\left(x^{*}-\mu_{2} B_{2} x^{*}\right)\right\|^{2} \\
& -\mu_{1}\left(2 \beta_{1}-\mu_{1}\right)\left\|B_{1} P_{C}\left(z_{n}-\mu_{2} B_{2} z_{n}\right)-B_{1} P_{C}\left(x^{*}-\mu_{2} B_{2} x^{*}\right)\right\|^{2} \\
\leq & \left\|\left(z_{n}-\mu_{2} B_{2} z_{n}\right)-\left(x^{*}-\mu_{2} B_{2} x^{*}\right)\right\|^{2}-\mu_{1}\left(2 \beta_{1}-\mu_{1}\right)\left\|B_{1} u_{n}-B_{1} y^{*}\right\|^{2} \\
= & \left\|\left(z_{n}-x^{*}\right)-\mu_{2}\left(B_{2} z_{n}-B_{2} x^{*}\right)\right\|^{2}-\mu_{1}\left(2 \beta_{1}-\mu_{1}\right)\left\|B_{1} u_{n}-B_{1} y^{*}\right\|^{2} \\
\leq & \left\|z_{n}-x^{*}\right\|^{2}-\mu_{2}\left(2 \beta_{2}-\mu_{2}\right)\left\|B_{2} z_{n}-B_{2} x^{*}\right\|^{2}-\mu_{1}\left(2 \beta_{1}-\mu_{1}\right)\left\|B_{1} u_{n}-B_{1} y^{*}\right\|^{2} \\
\leq & \left\|x_{n}-x^{*}\right\|^{2}-\lambda_{n}\left(2 \alpha-\lambda_{n}\right)\left\|A x_{n}-A x^{*}\right\|^{2} \\
& -\mu_{2}\left(2 \beta_{2}-\mu_{2}\right)\left\|B_{2} z_{n}-B_{2} x^{*}\right\|^{2}-\mu_{1}\left(2 \beta_{1}-\mu_{1}\right)\left\|B_{1} u_{n}-B_{1} y^{*}\right\|^{2} \\
\leq & \left\|x_{n}-x^{*}\right\|^{2} .
\end{aligned}
$$


Hence we get

$$
\begin{aligned}
\left\|y_{n}-x^{*}\right\| & =\left\|\alpha_{n}\left(Q x_{n}-x^{*}\right)+\left(1-\alpha_{n}\right)\left(P_{C}\left[P_{C}\left(z_{n}-\mu_{2} B_{2} z_{n}\right)-\mu_{1} B_{1} P_{C}\left(z_{n}-\mu_{2} B_{2} z_{n}\right)\right]-x^{*}\right)\right\| \\
& \leq \alpha_{n}\left\|Q x_{n}-x^{*}\right\|+\left(1-\alpha_{n}\right)\left\|P_{C}\left[P_{C}\left(z_{n}-\mu_{2} B_{2} z_{n}\right)-\mu_{1} B_{1} P_{C}\left(z_{n}-\mu_{2} B_{2} z_{n}\right)\right]-x^{*}\right\| \\
& \leq \alpha_{n}\left(\rho\left\|x_{n}-x^{*}\right\|+\left\|Q x^{*}-x^{*}\right\|\right)+\left(1-\alpha_{n}\right)\left\|x_{n}-x^{*}\right\| \\
& =\left(1-(1-\rho) \alpha_{n}\right)\left\|x_{n}-x^{*}\right\|+(1-\rho) \alpha_{n} \frac{\left\|Q x^{*}-x^{*}\right\|}{1-\rho} \\
& \leq \max \left\{\left\|x_{n}-x^{*}\right\|, \frac{\left\|Q x^{*}-x^{*}\right\|}{1-\rho}\right\} .
\end{aligned}
$$

Since $\left(\gamma_{n}+\delta_{n}\right) k \leq \gamma_{n}$ for all $n \geq 0$, utilizing Lemma 2.4 we obtain from (3.5)

$$
\begin{aligned}
\left\|x_{n+1}-x^{*}\right\| & =\left\|\beta_{n}\left(x_{n}-x^{*}\right)+\gamma_{n}\left(y_{n}-x^{*}\right)+\delta_{n}\left(S y_{n}-x^{*}\right)\right\| \\
& \leq \beta_{n}\left\|x_{n}-x^{*}\right\|+\left\|\gamma_{n}\left(y_{n}-x^{*}\right)+\delta_{n}\left(S y_{n}-x^{*}\right)\right\| \\
& \leq \beta_{n}\left\|x_{n}-x^{*}\right\|+\left(\gamma_{n}+\delta_{n}\right)\left\|y_{n}-x^{*}\right\| \\
& \leq \beta_{n}\left\|x_{n}-x^{*}\right\|+\left(\gamma_{n}+\delta_{n}\right) \max \left\{\left\|x_{n}-x^{*}\right\|, \frac{\left\|Q x^{*}-x^{*}\right\|}{1-\rho}\right\} \\
& \leq \max \left\{\left\|x_{n}-x^{*}\right\|, \frac{\left\|Q x^{*}-x^{*}\right\|}{1-\rho}\right\} .
\end{aligned}
$$

By induction, we obtain that for all $n \geq 0$

$$
\left\|x_{n}-x^{*}\right\| \leq \max \left\{\left\|x_{0}-x^{*}\right\|, \frac{\left\|Q x^{*}-x^{*}\right\|}{1-\rho}\right\}
$$

Hence, $\left\{x_{n}\right\}$ is bounded. Consequently, we deduce immediately that $\left\{z_{n}\right\},\left\{y_{n}\right\},\left\{S y_{n}\right\}$, and $\left\{u_{n}\right\}$ are bounded, where $u_{n}=P_{C}\left(z_{n}-\mu_{2} B_{2} z_{n}\right)$ for all $n \geq 0$.

Now, put

$$
t_{n}:=P_{C}\left[P_{C}\left(z_{n}-\mu_{2} B_{2} z_{n}\right)-\mu_{1} B_{1} P_{C}\left(z_{n}-\mu_{2} B_{2} z_{n}\right)\right], \quad \forall n \geq 0
$$

Then it is easy to see that $\left\{t_{n}\right\}$ is bounded because $P_{C}, B_{1}$, and $B_{2}$ are Lipschitz continuous and $\left\{z_{n}\right\}$ is bounded.

Step 2. $\lim _{n \rightarrow \infty}\left\|x_{n+1}-x_{n}\right\|=0$. 
Indeed, define $x_{n+1}=\beta_{n} x_{n}+\left(1-\beta_{n}\right) w_{n}$ for all $n \geq 0$. It follows that

$$
\begin{aligned}
w_{n+1}-w_{n}= & \frac{x_{n+2}-\beta_{n+1} x_{n+1}}{1-\beta_{n+1}}-\frac{x_{n+1}-\beta_{n} x_{n}}{1-\beta_{n}} \\
= & \frac{\gamma_{n+1} y_{n+1}+\delta_{n+1} S y_{n+1}}{1-\beta_{n+1}}-\frac{\gamma_{n} y_{n}+\delta_{n} S y_{n}}{1-\beta_{n}} \\
= & \frac{\gamma_{n+1}\left(y_{n+1}-y_{n}\right)+\delta_{n+1}\left(S y_{n+1}-S y_{n}\right)}{1-\beta_{n+1}}+\left(\frac{\gamma_{n+1}}{1-\beta_{n+1}}-\frac{\gamma_{n}}{1-\beta_{n}}\right) y_{n} \\
& +\left(\frac{\delta_{n+1}}{1-\beta_{n+1}}-\frac{\delta_{n}}{1-\beta_{n}}\right) S y_{n} .
\end{aligned}
$$

Since $\left(\gamma_{n}+\delta_{n}\right) k \leq \gamma_{n}$ for all $n \geq 0$, utilizing Lemma 2.4 we have

$$
\left\|\gamma_{n+1}\left(y_{n+1}-y_{n}\right)+\delta_{n+1}\left(S y_{n+1}-S y_{n}\right)\right\| \leq\left(\gamma_{n+1}+\delta_{n+1}\right)\left\|y_{n+1}-y_{n}\right\| .
$$

Next, we estimate $\left\|y_{n+1}-y_{n}\right\|$. Observe that

$$
\begin{aligned}
\left\|z_{n+1}-z_{n}\right\|= & \left\|P_{C}\left(x_{n+1}-\lambda_{n+1} A x_{n+1}\right)-P_{C}\left(x_{n}-\lambda_{n} A x_{n}\right)\right\| \\
\leq & \left\|\left(x_{n+1}-\lambda_{n+1} A x_{n+1}\right)-\left(x_{n}-\lambda_{n} A x_{n}\right)\right\| \\
= & \left\|\left(x_{n+1}-x_{n}\right)-\lambda_{n+1}\left(A x_{n+1}-A x_{n}\right)+\left(\lambda_{n}-\lambda_{n+1}\right) A x_{n}\right\| \\
\leq & \left\|\left(x_{n+1}-x_{n}\right)-\lambda_{n+1}\left(A x_{n+1}-A x_{n}\right)\right\|+\left|\lambda_{n+1}-\lambda_{n}\right|\left\|A x_{n}\right\| \\
\leq & \left\|x_{n+1}-x_{n}\right\|+\left|\lambda_{n+1}-\lambda_{n}\right|\left\|A x_{n}\right\|, \\
\left\|t_{n+1}-t_{n}\right\|^{2}= & \| P_{C}\left[P_{C}\left(z_{n+1}-\mu_{2} B_{2} z_{n+1}\right)-\mu_{1} B_{1} P_{C}\left(z_{n+1}-\mu_{2} B_{2} z_{n+1}\right)\right] \\
& -P_{C}\left[P_{C}\left(z_{n}-\mu_{2} B_{2} z_{n}\right)-\mu_{1} B_{1} P_{C}\left(z_{n}-\mu_{2} B_{2} z_{n}\right)\right] \|^{2} \\
\leq & \|\left[P_{C}\left(z_{n+1}-\mu_{2} B_{2} z_{n+1}\right)-\mu_{1} B_{1} P_{C}\left(z_{n+1}-\mu_{2} B_{2} z_{n+1}\right)\right] \\
& -\left[P_{C}\left(z_{n}-\mu_{2} B_{2} z_{n}\right)-\mu_{1} B_{1} P_{C}\left(z_{n}-\mu_{2} B_{2} z_{n}\right)\right] \|^{2} \\
= & \|\left[P_{C}\left(z_{n+1}-\mu_{2} B_{2} z_{n+1}\right)-P_{C}\left(z_{n}-\mu_{2} B_{2} z_{n}\right)\right] \\
& -\mu_{1}\left[B_{1} P_{C}\left(z_{n+1}-\mu_{2} B_{2} z_{n+1}\right)-B_{1} P_{C}\left(z_{n}-\mu_{2} B_{2} z_{n}\right)\right] \|^{2} \\
\leq & \left\|P_{C}\left(z_{n+1}-\mu_{2} B_{2} z_{n+1}\right)-P_{C}\left(z_{n}-\mu_{2} B_{2} z_{n}\right)\right\|^{2} \\
& -\mu_{1}\left(2 \beta_{1}-\mu_{1}\right)\left\|B_{1} P_{C}\left(z_{n+1}-\mu_{2} B_{2} z_{n+1}\right)-B_{1} P_{C}\left(z_{n}-\mu_{2} B_{2} z_{n}\right)\right\|^{2} \\
\leq & \left\|P_{C}\left(z_{n+1}-\mu_{2} B_{2} z_{n+1}\right)-P_{C}\left(z_{n}-\mu_{2} B_{2} z_{n}\right)\right\|^{2} \\
\leq & \left\|\left(z_{n+1}-\mu_{2} B_{2} z_{n+1}\right)-\left(z_{n}-\mu_{2} B_{2} z_{n}\right)\right\|^{2} \\
= & \left\|\left(z_{n+1}-z_{n}\right)-\mu_{2}\left(B_{2} z_{n+1}-B_{2} z_{n}\right)\right\|^{2} \\
\leq & \left\|z_{n+1}-z_{n}\right\|^{2}-\mu_{2}\left(2 \beta_{2}-\mu_{2}\right)\left\|B_{2} z_{n+1}-B_{2} z_{n}\right\|^{2} \\
\leq & \left\|z_{n+1}-z_{n}\right\|^{2} . \\
&
\end{aligned}
$$


Fixed Point Theory and Applications

Combining (3.11) with (3.12), we get

$$
\begin{aligned}
\left\|t_{n+1}-t_{n}\right\|= & \| P_{C}\left[P_{C}\left(z_{n+1}-\mu_{2} B_{2} z_{n+1}\right)-\mu_{1} B_{1} P_{C}\left(z_{n+1}-\mu_{2} B_{2} z_{n+1}\right)\right] \\
& -P_{C}\left[P_{C}\left(z_{n}-\mu_{2} B_{2} z_{n}\right)-\mu_{1} B_{1} P_{C}\left(z_{n}-\mu_{2} B_{2} z_{n}\right)\right] \| \\
\leq & \left\|x_{n+1}-x_{n}\right\|+\left|\lambda_{n+1}-\lambda_{n}\right|\left\|A x_{n}\right\| .
\end{aligned}
$$

This together with (3.13) implies that

$$
\begin{aligned}
\left\|y_{n+1}-y_{n}\right\| & =\left\|t_{n+1}+\alpha_{n+1}\left(Q x_{n+1}-t_{n+1}\right)-t_{n}-\alpha_{n}\left(Q x_{n}-t_{n}\right)\right\| \\
& \leq\left\|t_{n+1}-t_{n}\right\|+\alpha_{n+1}\left\|Q x_{n+1}-t_{n+1}\right\|+\alpha_{n}\left\|Q x_{n}-t_{n}\right\| \\
& \leq\left\|x_{n+1}-x_{n}\right\|+\left|\lambda_{n+1}-\lambda_{n}\right|\left\|A x_{n}\right\|+\alpha_{n+1}\left\|Q x_{n+1}-t_{n+1}\right\|+\alpha_{n}\left\|Q x_{n}-t_{n}\right\| .
\end{aligned}
$$

Hence it follows from (3.9), (3.10), and (3.14) that

$$
\begin{aligned}
\left\|w_{n+1}-w_{n}\right\| \leq & \frac{\left\|\gamma_{n+1}\left(y_{n+1}-y_{n}\right)+\delta_{n+1}\left(S y_{n+1}-S y_{n}\right)\right\|}{1-\beta_{n+1}} \\
& +\left|\frac{\gamma_{n+1}}{1-\beta_{n+1}}-\frac{\gamma_{n}}{1-\beta_{n}}\right|\left\|y_{n}\right\|+\left|\frac{\delta_{n+1}}{1-\beta_{n+1}}-\frac{\delta_{n}}{1-\beta_{n}}\right|\left\|S y_{n}\right\| \\
\leq & \frac{\gamma_{n+1}+\delta_{n+1}}{1-\beta_{n+1}}\left\|y_{n+1}-y_{n}\right\|+\left|\frac{\gamma_{n+1}}{1-\beta_{n+1}}-\frac{\gamma_{n}}{1-\beta_{n}}\right|\left(\left\|y_{n}\right\|+\left\|S y_{n}\right\|\right) \\
= & \left\|y_{n+1}-y_{n}\right\|+\left|\frac{\gamma_{n+1}}{1-\beta_{n+1}}-\frac{\gamma_{n}}{1-\beta_{n}}\right|\left(\left\|y_{n}\right\|+\left\|S y_{n}\right\|\right) \\
\leq & \left\|x_{n+1}-x_{n}\right\|+\left|\lambda_{n+1}-\lambda_{n}\right|\left\|A x_{n}\right\|+\alpha_{n+1}\left\|Q x_{n+1}-t_{n+1}\right\|+\alpha_{n}\left\|Q x_{n}-t_{n}\right\| \\
& +\left|\frac{\gamma_{n+1}}{1-\beta_{n+1}}-\frac{\gamma_{n}}{1-\beta_{n}}\right|\left(\left\|y_{n}\right\|+\left\|S y_{n}\right\|\right) .
\end{aligned}
$$

Since $\left\{x_{n}\right\},\left\{y_{n}\right\}$, and $\left\{t_{n}\right\}$ are bounded, it follows from conditions (ii), (iv), (v) that

$$
\begin{aligned}
& \limsup _{n \rightarrow \infty}\left(\left\|w_{n+1}-w_{n}\right\|-\left\|x_{n+1}-x_{n}\right\|\right) \\
& \leq \limsup _{n \rightarrow \infty}\left\{\left|\lambda_{n+1}-\lambda_{n}\right|\left\|A x_{n}\right\|+\alpha_{n+1}\left\|Q x_{n+1}-t_{n+1}\right\|+\alpha_{n}\left\|Q x_{n}-t_{n}\right\|\right. \\
& \left.\quad+\left|\frac{\gamma_{n+1}}{1-\beta_{n+1}}-\frac{\gamma_{n}}{1-\beta_{n}}\right|\left(\left\|y_{n}\right\|+\left\|S y_{n}\right\|\right)\right\}=0 .
\end{aligned}
$$

Hence by Lemma 2.1 we get $\lim _{n \rightarrow \infty}\left\|w_{n}-x_{n}\right\|=0$. Thus,

$$
\lim _{n \rightarrow \infty}\left\|x_{n+1}-x_{n}\right\|=\lim _{n \rightarrow \infty}\left(1-\beta_{n}\right)\left\|w_{n}-x_{n}\right\|=0 .
$$


Step 3. $\lim _{n \rightarrow \infty}\left\|B_{2} z_{n}-B_{2} x^{*}\right\|=0, \lim _{n \rightarrow \infty}\left\|B_{1} u_{n}-B_{1} y^{*}\right\|=0$ and $\lim _{n \rightarrow \infty}\left\|A x_{n}-A x^{*}\right\|=0$, where $y^{*}=P_{C}\left(x^{*}-\mu_{2} B_{2} x^{*}\right)$.

Indeed, utilizing Lemma 2.4 and the convexity of $\|\cdot\|^{2}$, we get from (3.1) and (3.4)

$$
\begin{aligned}
\left\|x_{n+1}-x^{*}\right\|^{2}= & \left\|\beta_{n}\left(x_{n}-x^{*}\right)+\gamma_{n}\left(y_{n}-x^{*}\right)+\delta_{n}\left(S y_{n}-x^{*}\right)\right\|^{2} \\
\leq & \beta_{n}\left\|x_{n}-x^{*}\right\|^{2}+\left(\gamma_{n}+\delta_{n}\right)\left\|\frac{1}{\gamma_{n}+\delta_{n}}\left[\gamma_{n}\left(y_{n}-x^{*}\right)+\delta_{n}\left(S y_{n}-x^{*}\right)\right]\right\|^{2} \\
\leq & \beta_{n}\left\|x_{n}-x^{*}\right\|^{2}+\left(\gamma_{n}+\delta_{n}\right)\left\|y_{n}-x^{*}\right\|^{2} \\
\leq & \beta_{n}\left\|x_{n}-x^{*}\right\|^{2}+\left(\gamma_{n}+\delta_{n}\right)\left[\alpha_{n}\left\|Q x_{n}-x^{*}\right\|^{2}+\left(1-\alpha_{n}\right)\left\|t_{n}-x^{*}\right\|^{2}\right] \\
\leq & \beta_{n}\left\|x_{n}-x^{*}\right\|^{2}+\alpha_{n}\left\|Q x_{n}-x^{*}\right\|^{2}+\left(\gamma_{n}+\delta_{n}\right)\left\|t_{n}-x^{*}\right\|^{2} \\
\leq & \beta_{n}\left\|x_{n}-x^{*}\right\|^{2}+\alpha_{n}\left\|Q x_{n}-x^{*}\right\|^{2}+\left(\gamma_{n}+\delta_{n}\right) \\
& \times\left[\left\|x_{n}-x^{*}\right\|^{2}-\lambda_{n}\left(2 \alpha-\lambda_{n}\right)\left\|A x_{n}-A x^{*}\right\|^{2}-\mu_{2}\left(2 \beta_{2}-\mu_{2}\right)\left\|B_{2} z_{n}-B_{2} x^{*}\right\|^{2}\right. \\
& \left.\quad-\mu_{1}\left(2 \beta_{1}-\mu_{1}\right)\left\|B_{1} u_{n}-B_{1} y^{*}\right\|^{2}\right] \\
= & \left\|x_{n}-x^{*}\right\|^{2}+\alpha_{n}\left\|Q x_{n}-x^{*}\right\|^{2}-\left(\gamma_{n}+\delta_{n}\right) \\
& \times\left[\lambda_{n}\left(2 \alpha-\lambda_{n}\right)\left\|A x_{n}-A x^{*}\right\|^{2}\right. \\
& \left.\quad-\mu_{2}\left(2 \beta_{2}-\mu_{2}\right)\left\|B_{2} z_{n}-B_{2} x^{*}\right\|^{2}-\mu_{1}\left(2 \beta_{1}-\mu_{1}\right)\left\|B_{1} u_{n}-B_{1} y^{*}\right\|^{2}\right] .
\end{aligned}
$$

Therefore,

$$
\begin{gathered}
\left(\gamma_{n}+\delta_{n}\right)\left[\lambda_{n}\left(2 \alpha-\lambda_{n}\right)\left\|A x_{n}-A x^{*}\right\|^{2}+\mu_{2}\left(2 \beta_{2}-\mu_{2}\right)\left\|B_{2} z_{n}-B_{2} x^{*}\right\|^{2}\right. \\
\left.+\mu_{1}\left(2 \beta_{1}-\mu_{1}\right)\left\|B_{1} u_{n}-B_{1} y^{*}\right\|^{2}\right] \\
\leq\left\|x_{n}-x^{*}\right\|^{2}-\left\|x_{n+1}-x^{*}\right\|^{2}+\alpha_{n}\left\|Q x_{n}-x^{*}\right\|^{2} \\
\leq\left(\left\|x_{n}-x^{*}\right\|+\left\|x_{n+1}-x^{*}\right\|\right)\left\|x_{n}-x_{n+1}\right\|+\alpha_{n}\left\|Q x_{n}-x^{*}\right\|^{2} .
\end{gathered}
$$

Since $\alpha_{n} \rightarrow 0,\left\|x_{n}-x_{n+1}\right\| \rightarrow 0, \liminf _{n \rightarrow \infty}\left(\gamma_{n}+\delta_{n}\right)>0$ and $0<\liminf _{n \rightarrow \infty} \lambda_{n} \leq \limsup _{n \rightarrow \infty} \lambda_{n}<$ $2 \alpha$, we have

$$
\lim _{n \rightarrow \infty}\left\|A x_{n}-A x^{*}\right\|=0, \quad \lim _{n \rightarrow \infty}\left\|B_{1} u_{n}-B_{1} y^{*}\right\|=0, \quad \lim _{n \rightarrow \infty}\left\|B_{2} z_{n}-B_{2} x^{*}\right\|=0 .
$$

Step 4. $\lim _{n \rightarrow \infty}\left\|S y_{n}-y_{n}\right\|=0$. 
Fixed Point Theory and Applications

Indeed, noticing the firm nonexpansivity of $P_{C}$ we have

$$
\begin{aligned}
\left\|z_{n}-x^{*}\right\|^{2}= & \left\|P_{C}\left(x_{n}-\lambda_{n} A x_{n}\right)-P_{C}\left(x^{*}-\lambda_{n} A x^{*}\right)\right\|^{2} \\
\leq & \left\langle\left(x_{n}-\lambda_{n} A x_{n}\right)-\left(x^{*}-\lambda_{n} A x^{*}\right), z_{n}-x^{*}\right\rangle \\
= & \frac{1}{2}\left[\left\|x_{n}-x^{*}-\lambda_{n}\left(A x_{n}-A x^{*}\right)\right\|^{2}+\left\|z_{n}-x^{*}\right\|^{2}\right. \\
& \left.\quad-\left\|\left(x_{n}-x^{*}\right)-\lambda_{n}\left(A x_{n}-A x^{*}\right)-\left(z_{n}-x^{*}\right)\right\|^{2}\right] \\
\leq & \frac{1}{2}\left[\left\|x_{n}-x^{*}\right\|^{2}+\left\|z_{n}-x^{*}\right\|^{2}-\left\|\left(x_{n}-z_{n}\right)-\lambda_{n}\left(A x_{n}-A x^{*}\right)\right\|^{2}\right] \\
= & \frac{1}{2}\left[\left\|x_{n}-x^{*}\right\|^{2}+\left\|z_{n}-x^{*}\right\|^{2}-\left\|x_{n}-z_{n}\right\|^{2}\right. \\
& \left.\quad+2 \lambda_{n}\left\langle x_{n}-z_{n}, A x_{n}-A x^{*}\right\rangle-\lambda_{n}^{2}\left\|A x_{n}-A x^{*}\right\|^{2}\right] \\
\leq & \frac{1}{2}\left[\left\|x_{n}-x^{*}\right\|^{2}+\left\|z_{n}-x^{*}\right\|^{2}-\left\|x_{n}-z_{n}\right\|^{2}+2 \lambda_{n}\left\|x_{n}-z_{n}\right\|\left\|A x_{n}-A x^{*}\right\|\right],
\end{aligned}
$$

that is,

$$
\left\|z_{n}-x^{*}\right\|^{2} \leq\left\|x_{n}-x^{*}\right\|^{2}-\left\|x_{n}-z_{n}\right\|^{2}+2 \lambda_{n}\left\|x_{n}-z_{n}\right\|\left\|A x_{n}-A x^{*}\right\| .
$$

Similarly to the above argument, we obtain

$$
\begin{aligned}
& \left\|u_{n}-y^{*}\right\|^{2}=\left\|P_{C}\left(z_{n}-\mu_{2} B_{2} z_{n}\right)-P_{C}\left(x^{*}-\mu_{2} B_{2} x^{*}\right)\right\|^{2} \\
& \leq\left\langle\left(z_{n}-\mu_{2} B_{2} z_{n}\right)-\left(x^{*}-\mu_{2} B_{2} x^{*}\right), u_{n}-y^{*}\right\rangle \\
& =\frac{1}{2}\left[\left\|z_{n}-x^{*}-\mu_{2}\left(B_{2} z_{n}-B_{2} x^{*}\right)\right\|^{2}+\left\|u_{n}-y^{*}\right\|^{2}\right. \\
& \left.-\left\|\left(z_{n}-x^{*}\right)-\mu_{2}\left(B_{2} z_{n}-B_{2} x^{*}\right)-\left(u_{n}-y^{*}\right)\right\|^{2}\right] \\
& \leq \frac{1}{2}\left[\left\|z_{n}-x^{*}\right\|^{2}+\left\|u_{n}-y^{*}\right\|^{2}-\left\|\left(z_{n}-u_{n}\right)-\mu_{2}\left(B_{2} z_{n}-B_{2} x^{*}\right)-\left(x^{*}-y^{*}\right)\right\|^{2}\right] \\
& =\frac{1}{2}\left[\left\|z_{n}-x^{*}\right\|^{2}+\left\|u_{n}-y^{*}\right\|^{2}-\left\|z_{n}-u_{n}-\left(x^{*}-y^{*}\right)\right\|^{2}\right. \\
& \left.+2 \mu_{2}\left\langle z_{n}-u_{n}-\left(x^{*}-y^{*}\right), B_{2} z_{n}-B_{2} x^{*}\right\rangle-\mu_{2}^{2}\left\|B_{2} z_{n}-B_{2} x^{*}\right\|^{2}\right],
\end{aligned}
$$

that is,

$$
\left\|u_{n}-y^{*}\right\|^{2} \leq\left\|z_{n}-x^{*}\right\|^{2}-\left\|z_{n}-u_{n}-\left(x^{*}-y^{*}\right)\right\|^{2}+2 \mu_{2}\left\|z_{n}-u_{n}-\left(x^{*}-y^{*}\right)\right\|\left\|B_{2} z_{n}-B_{2} x^{*}\right\| .
$$


Substituting (3.22) in (3.24), we have

$$
\begin{aligned}
\left\|u_{n}-y^{*}\right\|^{2} \leq & \left\|x_{n}-x^{*}\right\|^{2}-\left\|x_{n}-z_{n}\right\|^{2}+2 \lambda_{n}\left\|x_{n}-z_{n}\right\|\left\|A x_{n}-A x^{*}\right\| \\
& -\left\|z_{n}-u_{n}-\left(x^{*}-y^{*}\right)\right\|^{2}+2 \mu_{2}\left\|z_{n}-u_{n}-\left(x^{*}-y^{*}\right)\right\|\left\|B_{2} z_{n}-B_{2} x^{*}\right\| .
\end{aligned}
$$

Further, similarly to the above argument, we derive

$$
\begin{aligned}
\left\|t_{n}-x^{*}\right\|^{2}= & \left\|P_{C}\left(u_{n}-\mu_{1} B_{1} u_{n}\right)-P_{C}\left(y^{*}-\mu_{1} B_{1} y^{*}\right)\right\|^{2} \\
\leq & \left\langle\left(u_{n}-\mu_{1} B_{1} u_{n}\right)-\left(y^{*}-\mu_{1} B_{1} y^{*}\right), t_{n}-x^{*}\right\rangle \\
= & \frac{1}{2}\left[\left\|u_{n}-y^{*}-\mu_{1}\left(B_{1} u_{n}-B_{1} y^{*}\right)\right\|^{2}+\left\|t_{n}-x^{*}\right\|^{2}\right. \\
& \left.\quad\left\|\left(u_{n}-y^{*}\right)-\mu_{1}\left(B_{1} u_{n}-B_{1} y^{*}\right)-\left(t_{n}-x^{*}\right)\right\|^{2}\right] \\
\leq & \frac{1}{2}\left[\left\|u_{n}-y^{*}\right\|^{2}+\left\|t_{n}-x^{*}\right\|^{2}-\left\|\left(u_{n}-t_{n}\right)-\mu_{1}\left(B_{1} u_{n}-B_{1} y^{*}\right)+\left(x^{*}-y^{*}\right)\right\|^{2}\right] \\
= & \frac{1}{2}\left[\left\|u_{n}-y^{*}\right\|^{2}+\left\|t_{n}-x^{*}\right\|^{2}-\left\|u_{n}-t_{n}+\left(x^{*}-y^{*}\right)\right\|^{2}\right. \\
& \left.\quad+2 \mu_{1}\left\langle u_{n}-t_{n}+\left(x^{*}-y^{*}\right), B_{1} u_{n}-B_{1} y^{*}\right\rangle-\mu_{1}^{2}\left\|B_{1} u_{n}-B_{1} y^{*}\right\|^{2}\right],
\end{aligned}
$$

that is,

$$
\left\|t_{n}-x^{*}\right\|^{2} \leq\left\|u_{n}-y^{*}\right\|^{2}-\left\|u_{n}-t_{n}+\left(x^{*}-y^{*}\right)\right\|^{2}+2 \mu_{1}\left\|u_{n}-t_{n}+\left(x^{*}-y^{*}\right)\right\|\left\|B_{1} u_{n}-B_{1} y^{*}\right\| .
$$

Substituting (3.25) in (3.27), we have

$$
\begin{aligned}
\left\|t_{n}-x^{*}\right\|^{2} \leq & \left\|x_{n}-x^{*}\right\|^{2}-\left\|x_{n}-z_{n}\right\|^{2}+2 \lambda_{n}\left\|x_{n}-z_{n}\right\|\left\|A x_{n}-A x^{*}\right\| \\
& -\left\|z_{n}-u_{n}-\left(x^{*}-y^{*}\right)\right\|^{2}+2 \mu_{2}\left\|z_{n}-u_{n}-\left(x^{*}-y^{*}\right)\right\|\left\|B_{2} z_{n}-B_{2} x^{*}\right\| \\
& -\left\|u_{n}-t_{n}+\left(x^{*}-y^{*}\right)\right\|^{2}+2 \mu_{1}\left\|u_{n}-t_{n}+\left(x^{*}-y^{*}\right)\right\|\left\|B_{1} u_{n}-B_{1} y^{*}\right\| .
\end{aligned}
$$


Fixed Point Theory and Applications

Thus from (3.1) and (3.28), it follows that

$$
\begin{aligned}
\left\|x_{n+1}-x^{*}\right\|^{2}= & \left\|\beta_{n}\left(x_{n}-x^{*}\right)+\gamma_{n}\left(y_{n}-x^{*}\right)+\delta_{n}\left(S y_{n}-x^{*}\right)\right\|^{2} \\
\leq & \beta_{n}\left\|x_{n}-x^{*}\right\|^{2}+\left(\gamma_{n}+\delta_{n}\right)\left\|y_{n}-x^{*}\right\|^{2} \\
= & \beta_{n}\left\|x_{n}-x^{*}\right\|^{2}+\left(1-\beta_{n}\right)\left\|y_{n}-x^{*}\right\|^{2} \\
\leq & \beta_{n}\left\|x_{n}-x^{*}\right\|^{2}+\left(1-\beta_{n}\right)\left[\alpha_{n}\left\|Q x_{n}-x^{*}\right\|^{2}+\left(1-\alpha_{n}\right)\left\|t_{n}-x^{*}\right\|^{2}\right] \\
\leq & \beta_{n}\left\|x_{n}-x^{*}\right\|^{2}+\alpha_{n}\left\|Q x_{n}-x^{*}\right\|^{2}+\left(1-\beta_{n}\right)\left\|t_{n}-x^{*}\right\|^{2} \\
\leq & \beta_{n}\left\|x_{n}-x^{*}\right\|^{2}+\alpha_{n}\left\|Q x_{n}-x^{*}\right\|^{2}+\left(1-\beta_{n}\right) \\
& \times\left[\left\|x_{n}-x^{*}\right\|^{2}-\left\|x_{n}-z_{n}\right\|^{2}+2 \lambda_{n}\left\|x_{n}-z_{n}\right\|\left\|A x_{n}-A x^{*}\right\|\right. \\
& \quad-\left\|z_{n}-u_{n}-\left(x^{*}-y^{*}\right)\right\|^{2}+2 \mu_{2}\left\|z_{n}-u_{n}-\left(x^{*}-y^{*}\right)\right\|\left\|B_{2} z_{n}-B_{2} x^{*}\right\| \\
& \left.\quad-\left\|u_{n}-t_{n}+\left(x^{*}-y^{*}\right)\right\|^{2}+2 \mu_{1}\left\|u_{n}-t_{n}+\left(x^{*}-y^{*}\right)\right\|\left\|B_{1} u_{n}-B_{1} y^{*}\right\|\right] \\
= & \left\|x_{n}-x^{*}\right\|^{2}+\alpha_{n}\left\|Q x_{n}-x^{*}\right\|^{2}+\left(1-\beta_{n}\right) \\
& \times\left[2 \lambda_{n}\left\|x_{n}-z_{n}\right\|\left\|A x_{n}-A x^{*}\right\|+2 \mu_{2}\left\|z_{n}-u_{n}-\left(x^{*}-y^{*}\right)\right\|\left\|B_{2} z_{n}-B_{2} x^{*}\right\|\right. \\
& \left.+2 \mu_{1}\left\|u_{n}-t_{n}+\left(x^{*}-y^{*}\right)\right\|\left\|B_{1} u_{n}-B_{1} y^{*}\right\|\right] \\
& -\left(1-\beta_{n}\right)\left[\left\|x_{n}-z_{n}\right\|^{2}+\left\|z_{n}-u_{n}-\left(x^{*}-y^{*}\right)\right\|^{2}+\left\|u_{n}-t_{n}+\left(x^{*}-y^{*}\right)\right\|^{2}\right],
\end{aligned}
$$

which hence implies that

$$
\begin{aligned}
\left(1-\beta_{n}\right)[ & \left.\left\|x_{n}-z_{n}\right\|^{2}+\left\|z_{n}-u_{n}-\left(x^{*}-y^{*}\right)\right\|^{2}+\left\|u_{n}-t_{n}+\left(x^{*}-y^{*}\right)\right\|^{2}\right] \\
\leq & \left\|x_{n}-x^{*}\right\|^{2}-\left\|x_{n+1}-x^{*}\right\|^{2}+\alpha_{n}\left\|Q x_{n}-x^{*}\right\|^{2}+\left(1-\beta_{n}\right) \\
\times & {\left[2 \lambda_{n}\left\|x_{n}-z_{n}\right\|\left\|A x_{n}-A x^{*}\right\|+2 \mu_{2}\left\|z_{n}-u_{n}-\left(x^{*}-y^{*}\right)\right\|\left\|B_{2} z_{n}-B_{2} x^{*}\right\|\right.} \\
& \left.+2 \mu_{1}\left\|u_{n}-t_{n}+\left(x^{*}-y^{*}\right)\right\|\left\|B_{1} u_{n}-B_{1} y^{*}\right\|\right] \\
\leq & \left(\left\|x_{n}-x^{*}\right\|+\left\|x_{n+1}-x^{*}\right\|\right)\left\|x_{n}-x_{n+1}\right\|+\alpha_{n}\left\|Q x_{n}-x^{*}\right\|^{2}+\left(1-\beta_{n}\right) \\
\times & {\left[2 \lambda_{n}\left\|x_{n}-z_{n}\right\|\left\|A x_{n}-A x^{*}\right\|+2 \mu_{2}\left\|z_{n}-u_{n}-\left(x^{*}-y^{*}\right)\right\|\left\|B_{2} z_{n}-B_{2} x^{*}\right\|\right.} \\
& \left.+2 \mu_{1}\left\|u_{n}-t_{n}+\left(x^{*}-y^{*}\right)\right\|\left\|B_{1} u_{n}-B_{1} y^{*}\right\|\right] .
\end{aligned}
$$


Since $\limsup \sup _{n \rightarrow \infty} \beta_{n}<1,0<\lambda_{n} \leq 2 \alpha, \alpha_{n} \rightarrow 0,\left\|A x_{n}-A x^{*}\right\| \rightarrow 0,\left\|B_{2} z_{n}-B_{2} x^{*}\right\| \rightarrow 0$, $\left\|B_{1} u_{n}-B_{1} y^{*}\right\| \rightarrow 0$ and $\left\|x_{n+1}-x_{n}\right\| \rightarrow 0$, it follows from the boundedness of $\left\{x_{n}\right\},\left\{z_{n}\right\},\left\{u_{n}\right\}$, and $\left\{t_{n}\right\}$ that

$$
\lim _{n \rightarrow \infty}\left\|x_{n}-z_{n}\right\|=0, \quad \lim _{n \rightarrow \infty}\left\|z_{n}-u_{n}-\left(x^{*}-y^{*}\right)\right\|=0, \quad \lim _{n \rightarrow \infty}\left\|u_{n}-t_{n}+\left(x^{*}-y^{*}\right)\right\|=0 .
$$

Consequently, it immediately follows that

$$
\lim _{n \rightarrow \infty}\left\|z_{n}-t_{n}\right\|=0, \quad \lim _{n \rightarrow \infty}\left\|x_{n}-t_{n}\right\|=0
$$

This together with $\left\|y_{n}-t_{n}\right\| \leq \alpha_{n}\left\|Q x_{n}-t_{n}\right\| \rightarrow 0$ implies that

$$
\lim _{n \rightarrow \infty}\left\|x_{n}-y_{n}\right\|=0
$$

Since

$$
\left\|\delta_{n}\left(S y_{n}-x_{n}\right)\right\| \leq\left\|x_{n+1}-x_{n}\right\|+\gamma_{n}\left\|y_{n}-x_{n}\right\|
$$

it follows that

$$
\lim _{n \rightarrow \infty}\left\|S y_{n}-x_{n}\right\|=0, \quad \lim _{n \rightarrow \infty}\left\|S y_{n}-y_{n}\right\|=0
$$

Step 5. $\lim \sup _{n \rightarrow \infty}\left\langle Q \bar{x}-\bar{x}, x_{n}-\bar{x}\right\rangle \leq 0$, where $\bar{x}=P_{\mathrm{Fix}(S) \cap \Gamma \cap \mathrm{VI}(A, C)} \cdot Q \bar{x}$.

Indeed, since $\left\{x_{n}\right\}$ is bounded, there exists a subsequence $\left\{x_{n_{i}}\right\}$ of $\left\{x_{n}\right\}$ such that

$$
\limsup _{n \rightarrow \infty}\left\langle Q \bar{x}-\bar{x}, x_{n}-\bar{x}\right\rangle=\lim _{i \rightarrow \infty}\left\langle Q \bar{x}-\bar{x}, x_{n_{i}}-\bar{x}\right\rangle
$$

Also, since $H$ is reflexive and $\left\{y_{n}\right\}$ is bounded, without loss of generality we may assume that $y_{n_{i}} \rightarrow p$ weakly for some $p \in C$. First, it is clear from Lemma 2.2 that $p \in \operatorname{Fix}(S)$. Now let us show that $p \in \Gamma$. We note that

$$
\begin{aligned}
& \left\|y_{n}-G\left(y_{n}\right)\right\| \\
& \leq \alpha_{n}\left\|Q x_{n}-G\left(y_{n}\right)\right\|+\left(1-\alpha_{n}\right)\left\|P_{C}\left[P_{C}\left(z_{n}-\mu_{2} B_{2} z_{n}\right)-\mu_{1} B_{1} P_{C}\left(z_{n}-\mu_{2} B_{2} z_{n}\right)\right]-G\left(y_{n}\right)\right\| \\
& =\alpha_{n}\left\|Q x_{n}-G\left(y_{n}\right)\right\|+\left(1-\alpha_{n}\right)\left\|G\left(z_{n}\right)-G\left(y_{n}\right)\right\| \\
& \leq \alpha_{n}\left\|Q x_{n}-G\left(y_{n}\right)\right\|+\left(1-\alpha_{n}\right)\left\|x_{n}-y_{n}\right\| \\
& \longrightarrow 0 .
\end{aligned}
$$


According to Lemma 2.2 we obtain $p \in \Gamma$. Further, let us show that $p \in \operatorname{VI}(A, C)$. As a matter of fact, since $\left\|x_{n}-z_{n}\right\| \rightarrow 0$ and $\left\|x_{n}-y_{n}\right\| \rightarrow 0$, we deduce that $x_{n_{i}} \rightarrow p$ weakly and $z_{n_{i}} \rightarrow p$ weakly. Let

$$
T v= \begin{cases}A v+N_{C} v & \text { if } v \in C, \\ \emptyset & \text { if } v \notin C,\end{cases}
$$

where $N_{C} v$ is the normal cone to $C$ at $v \in C$. In this case, the mapping $T$ is maximal monotone, and $0 \in T v$ if and only if $v \in \operatorname{VI}(A, C)$; see [10] for more details. Let $\operatorname{Gph}(T)$ be the graph of $T$ and let $(v, w) \in \operatorname{Gph}(T)$. Then, we have $w \in T v=A v+N_{C} v$ and hence $w-A v \in N_{C} v$. So, we have $\langle v-t, w-A v\rangle \geq 0$ for all $t \in C$. On the other hand, from $z_{n}=P_{C}\left(x_{n}-\lambda_{n} A x_{n}\right)$ and $v \in C$ we have

$$
\left\langle x_{n}-\lambda_{n} A x_{n}-z_{n}, z_{n}-v\right\rangle \geq 0
$$

and hence

$$
\left\langle v-z_{n}, \frac{z_{n}-x_{n}}{\lambda_{n}}+A x_{n}\right\rangle \geq 0
$$

From $\langle v-t, w-A v\rangle \geq 0$ for all $t \in C$ and $z_{n_{i}} \in C$, we have

$$
\begin{aligned}
\left\langle v-z_{n_{i}}, w\right\rangle & \geq\left\langle v-z_{n_{i}}, A v\right\rangle \\
& \geq\left\langle v-z_{n_{i}}, A v\right\rangle-\left\langle v-z_{n_{i}}, \frac{z_{n_{i}}-x_{n_{i}}}{\lambda_{n_{i}}}+A x_{n_{i}}\right\rangle \\
& =\left\langle v-z_{n_{i}}, A v-A z_{n_{i}}\right\rangle+\left\langle v-z_{n_{i}}, A z_{n_{i}}-A x_{n_{i}}\right\rangle-\left\langle v-z_{n_{i}}, \frac{z_{n_{i}}-x_{n_{i}}}{\lambda_{n_{i}}}\right\rangle \\
& \geq\left\langle v-z_{n_{i}}, A z_{n_{i}}-A x_{n_{i}}\right\rangle-\left\langle v-z_{n_{i}}, \frac{z_{n_{i}}-x_{n_{i}}}{\lambda_{n_{i}}}\right\rangle
\end{aligned}
$$

Hence, we obtain $\langle v-p, w\rangle \geq 0$ as $i \rightarrow \infty$. Since $T$ is maximal monotone, we have $p \in T^{-1} 0$ and hence $p \in \operatorname{VI}(A, C)$. Therefore, $p \in \operatorname{Fix}(S) \cap \Gamma \cap \operatorname{VI}(A, C)$. Hence it follows from (2.8) and (3.36) that

$$
\begin{aligned}
\limsup _{n \rightarrow \infty}\left\langle Q \bar{x}-\bar{x}, x_{n}-\bar{x}\right\rangle & =\lim _{i \rightarrow \infty}\left\langle Q \bar{x}-\bar{x}, x_{n_{i}}-\bar{x}\right\rangle \\
& =\langle Q \bar{x}-\bar{x}, p-\bar{x}\rangle \\
& \leq 0 .
\end{aligned}
$$

Step 6. $\lim _{n \rightarrow \infty} x_{n}=\bar{x}$.

Indeed, since $G: C \rightarrow C$ is nonexpansive, we have

$$
\left\|t_{n}-\bar{x}\right\|=\left\|G\left(z_{n}\right)-G(\bar{x})\right\| \leq\left\|x_{n}-\bar{x}\right\| .
$$


Note that

$$
\begin{aligned}
\left\langle Q x_{n}-\bar{x}, y_{n}-\bar{x}\right\rangle & =\left\langle Q x_{n}-\bar{x}, x_{n}-\bar{x}\right\rangle+\left\langle Q x_{n}-\bar{x}, y_{n}-x_{n}\right\rangle \\
& =\left\langle Q x_{n}-Q \bar{x}, x_{n}-\bar{x}\right\rangle+\left\langle Q \bar{x}-\bar{x}, x_{n}-\bar{x}\right\rangle+\left\langle Q x_{n}-\bar{x}, y_{n}-x_{n}\right\rangle \\
& \leq \rho\left\|x_{n}-\bar{x}\right\|^{2}+\left\langle Q \bar{x}-\bar{x}, x_{n}-\bar{x}\right\rangle+\left\|Q x_{n}-\bar{x}\right\|\left\|y_{n}-x_{n}\right\| .
\end{aligned}
$$

Utilizing Lemmas 2.4 and 2.5, we obtain from (3.4) and the convexity of $\|\cdot\|^{2}$

$$
\begin{aligned}
\left\|x_{n+1}-\bar{x}\right\|^{2}= & \left\|\beta_{n}\left(x_{n}-\bar{x}\right)+\gamma_{n}\left(y_{n}-\bar{x}\right)+\delta_{n}\left(S y_{n}-\bar{x}\right)\right\|^{2} \\
\leq & \beta_{n}\left\|x_{n}-\bar{x}\right\|^{2}+\left(\gamma_{n}+\delta_{n}\right)\left\|\frac{1}{\gamma_{n}+\delta_{n}}\left[\gamma_{n}\left(y_{n}-\bar{x}\right)+\delta_{n}\left(S y_{n}-\bar{x}\right)\right]\right\|^{2} \\
\leq & \beta_{n}\left\|x_{n}-\bar{x}\right\|^{2}+\left(\gamma_{n}+\delta_{n}\right)\left\|y_{n}-\bar{x}\right\|^{2} \\
\leq & \beta_{n}\left\|x_{n}-\bar{x}\right\|^{2}+\left(\gamma_{n}+\delta_{n}\right)\left[\left(1-\alpha_{n}\right)^{2}\left\|t_{n}-\bar{x}\right\|^{2}+2 \alpha_{n}\left\langle Q x_{n}-\bar{x}, y_{n}-\bar{x}\right\rangle\right] \\
\leq & \beta_{n}\left\|x_{n}-\bar{x}\right\|^{2}+\left(\gamma_{n}+\delta_{n}\right)\left[\left(1-\alpha_{n}\right)\left\|x_{n}-\bar{x}\right\|^{2}+2 \alpha_{n}\left\langle Q x_{n}-\bar{x}, y_{n}-\bar{x}\right\rangle\right] \\
= & \left(1-\left(\gamma_{n}+\delta_{n}\right) \alpha_{n}\right)\left\|x_{n}-\bar{x}\right\|^{2}+\left(\gamma_{n}+\delta_{n}\right) 2 \alpha_{n}\left\langle Q x_{n}-\bar{x}, y_{n}-\bar{x}\right\rangle \\
\leq & \left(1-\left(\gamma_{n}+\delta_{n}\right) \alpha_{n}\right)\left\|x_{n}-\bar{x}\right\|^{2} \\
& +\left(\gamma_{n}+\delta_{n}\right) 2 \alpha_{n}\left[\rho\left\|x_{n}-\bar{x}\right\|^{2}+\left\langle Q \bar{x}-\bar{x}, x_{n}-\bar{x}\right\rangle+\left\|Q x_{n}-\bar{x}\right\|\left\|y_{n}-x_{n}\right\|\right] \\
\leq & {\left[1-(1-2 \rho)\left(\gamma_{n}+\delta_{n}\right) \alpha_{n}\right]\left\|x_{n}-\bar{x}\right\|^{2} } \\
& +\left(\gamma_{n}+\delta_{n}\right) 2 \alpha_{n}\left[\left\langle Q \bar{x}-\bar{x}, x_{n}-\bar{x}\right\rangle+\left\|Q x_{n}-\bar{x}\right\|\left\|y_{n}-x_{n}\right\|\right] \\
= & {\left[1-(1-2 \rho)\left(\gamma_{n}+\delta_{n}\right) \alpha_{n}\right]\left\|x_{n}-\bar{x}\right\|^{2} } \\
& +(1-2 \rho)\left(\gamma_{n}+\delta_{n}\right) \alpha_{n} \frac{2\left[\left\langle Q \bar{x}-\bar{x}, x_{n}-\bar{x}\right\rangle+\left\|Q x_{n}-\bar{x}\right\|\left\|y_{n}-x_{n}\right\|\right]}{1-2 \rho} . \\
& +(1-x)
\end{aligned}
$$

Note that $\liminf _{n \rightarrow \infty}(1-2 \rho)\left(\gamma_{n}+\delta_{n}\right)>0$. It follows that $\sum_{n=0}^{\infty}(1-2 \rho)\left(\gamma_{n}+\delta_{n}\right) \alpha_{n}=\infty$. It is clear that

$$
\limsup _{n \rightarrow \infty} \frac{2\left[\left\langle Q \bar{x}-\bar{x}, x_{n}-\bar{x}\right\rangle+\left\|Q x_{n}-\bar{x}\right\|\left\|y_{n}-x_{n}\right\|\right]}{1-2 \rho} \leq 0
$$

because $\limsup _{n \rightarrow \infty}\left\langle Q \bar{x}-\bar{x}, x_{n}-\bar{x}\right\rangle \leq 0$ and $\lim _{n \rightarrow \infty}\left\|x_{n}-y_{n}\right\|=0$. Therefore, all conditions of Lemma 2.3 are satisfied. Consequently, we immediately deduce that $x_{n} \rightarrow \bar{x}$. This completes the proof.

Corollary 3.2. Let $C$ be a nonempty closed convex subset of a real Hilbert space $H$. Let $A: C \rightarrow H$ be $\alpha$-inverse strongly monotone and $B_{i}: C \rightarrow H$ be $\beta_{i}$-inverse strongly monotone for $i=1,2$. Let 
$S: C \rightarrow C$ be a $k$-strictly pseudocontractive mapping such that $\operatorname{Fix}(S) \cap \Gamma \cap V I(A, C) \neq \emptyset$. For fixed $u \in C$ and given $x_{0} \in C$ arbitrarily, let the sequences $\left\{x_{n}\right\},\left\{y_{n}\right\}$, and $\left\{z_{n}\right\}$ be generated iteratively by

$$
\begin{gathered}
z_{n}=P_{C}\left(x_{n}-\lambda_{n} A x_{n}\right), \\
y_{n}=\alpha_{n} u+\left(1-\alpha_{n}\right) P_{C}\left[P_{C}\left(z_{n}-\mu_{2} B_{2} z_{n}\right)-\mu_{1} B_{1} P_{C}\left(z_{n}-\mu_{2} B_{2} z_{n}\right)\right], \\
x_{n+1}=\beta_{n} x_{n}+\gamma_{n} y_{n}+\delta_{n} S y_{n}, \quad \forall n \geq 0,
\end{gathered}
$$

where $\mu_{i} \in\left(0,2 \beta_{i}\right)$ for $i=1,2,\left\{\lambda_{n}\right\} \subset(0,2 \alpha]$ and $\left\{\alpha_{n}\right\},\left\{\beta_{n}\right\},\left\{\gamma_{n}\right\},\left\{\delta_{n}\right\} \subset[0,1]$ such that

(i) $\beta_{n}+\gamma_{n}+\delta_{n}=1$ and $\left(\gamma_{n}+\delta_{n}\right) k \leq \gamma_{n}$ for all $n \geq 0$;

(ii) $\lim _{n \rightarrow \infty} \alpha_{n}=0$ and $\sum_{n=0}^{\infty} \alpha_{n}=\infty$;

(iii) $0<\liminf _{n \rightarrow \infty} \beta_{n} \leq \limsup _{n \rightarrow \infty} \beta_{n}<1$ and $\liminf _{n \rightarrow \infty} \delta_{n}>0$;

(iv) $\lim _{n \rightarrow \infty}\left(\gamma_{n+1} /\left(1-\beta_{n+1}\right)-\gamma_{n} /\left(1-\beta_{n}\right)\right)=0$;

(v) $0<\liminf _{n \rightarrow \infty} \lambda_{n} \leq \limsup _{n \rightarrow \infty} \lambda_{n}<2 \alpha$ and $\lim _{n \rightarrow \infty}\left|\lambda_{n+1}-\lambda_{n}\right|=0$.

Then the sequence $\left\{x_{n}\right\}$ converges strongly to $\bar{x}=P_{\mathrm{Fix}(S) \cap \Gamma \cap V I(A, C)} \cdot Q \bar{x}$ and $(\bar{x}, \bar{y})$ is a solution of the general system (1.3) of variational inequalities, where $\bar{y}=P_{C}\left(\bar{x}-\mu_{2} B_{2} \bar{x}\right)$.

Corollary 3.3. Let $C$ be a nonempty closed convex subset of a real Hilbert space $H$. Let $A: C \rightarrow H$ be $\alpha$-inverse strongly monotone and $B_{i}: C \rightarrow H$ be $\beta_{i}$-inverse strongly monotone for $i=1,2$. Let $S: C \rightarrow C$ be a nonexpansive mapping such that $\operatorname{Fix}(S) \cap \Gamma \cap V I(A, C) \neq \emptyset$. Let $Q: C \rightarrow C$ be a $\rho$-contraction with $\rho \in[0,1 / 2)$. For given $x_{0} \in C$ arbitrarily, let the sequences $\left\{x_{n}\right\},\left\{y_{n}\right\}$ and $\left\{z_{n}\right\}$ be generated iteratively by

$$
\begin{gathered}
z_{n}=P_{C}\left(x_{n}-\lambda_{n} A x_{n}\right), \\
y_{n}=\alpha_{n} Q x_{n}+\left(1-\alpha_{n}\right) P_{C}\left[P_{C}\left(z_{n}-\mu_{2} B_{2} z_{n}\right)-\mu_{1} B_{1} P_{C}\left(z_{n}-\mu_{2} B_{2} z_{n}\right)\right], \\
x_{n+1}=\beta_{n} x_{n}+\gamma_{n} y_{n}+\delta_{n} S y_{n}, \quad \forall n \geq 0,
\end{gathered}
$$

where $\mu_{i} \in\left(0,2 \beta_{i}\right)$ for $i=1,2,\left\{\lambda_{n}\right\} \subset(0,2 \alpha]$ and $\left\{\alpha_{n}\right\},\left\{\beta_{n}\right\},\left\{\gamma_{n}\right\},\left\{\delta_{n}\right\} \subset[0,1]$ such that

(i) $\beta_{n}+\gamma_{n}+\delta_{n}=1$ for all $n \geq 0$;

(ii) $\lim _{n \rightarrow \infty} \alpha_{n}=0$ and $\sum_{n=0}^{\infty} \alpha_{n}=\infty$;

(iii) $0<\liminf _{n \rightarrow \infty} \beta_{n} \leq \limsup _{n \rightarrow \infty} \beta_{n}<1$ and $\liminf _{n \rightarrow \infty} \delta_{n}>0$;

(iv) $\lim _{n \rightarrow \infty}\left(\gamma_{n+1} /\left(1-\beta_{n+1}\right)-\gamma_{n} /\left(1-\beta_{n}\right)\right)=0$;

(v) $0<\liminf _{n \rightarrow \infty} \lambda_{n} \leq \limsup _{n \rightarrow \infty} \lambda_{n}<2 \alpha$ and $\lim _{n \rightarrow \infty}\left|\lambda_{n+1}-\lambda_{n}\right|=0$.

Then the sequence $\left\{x_{n}\right\}$ converges strongly to $\bar{x}=P_{\operatorname{Fix}(S) \cap \Gamma \cap V I(A, C)} \cdot Q \bar{x}$ and $(\bar{x}, \bar{y})$ is a solution of the general system (1.3) of variational inequalities, where $\bar{y}=P_{C}\left(\bar{x}-\mu_{2} B_{2} \bar{x}\right)$.

Corollary 3.4. Let $C$ be a nonempty closed convex subset of a real Hilbert space $H$. Let $A: C \rightarrow H$ be $\alpha$-inverse strongly monotone and $B_{i}: C \rightarrow H$ be $\beta_{i}$-inverse strongly monotone for $i=1,2$. Let 
$S: C \rightarrow C$ be a nonexpansive mapping such that $\operatorname{Fix}(S) \cap \Gamma \cap V I(A, C) \neq \emptyset$. For fixed $u \in C$ and given $x_{0} \in C$ arbitrarily, let the sequences $\left\{x_{n}\right\},\left\{y_{n}\right\}$ and $\left\{z_{n}\right\}$ be generated iteratively by

$$
\begin{gathered}
z_{n}=P_{C}\left(x_{n}-\lambda_{n} A x_{n}\right), \\
y_{n}=\alpha_{n} u+\left(1-\alpha_{n}\right) P_{C}\left[P_{C}\left(z_{n}-\mu_{2} B_{2} z_{n}\right)-\mu_{1} B_{1} P_{C}\left(z_{n}-\mu_{2} B_{2} z_{n}\right)\right], \\
x_{n+1}=\beta_{n} x_{n}+\gamma_{n} y_{n}+\delta_{n} S y_{n}, \quad \forall n \geq 0,
\end{gathered}
$$

where $\mu_{i} \in\left(0,2 \beta_{i}\right)$ for $i=1,2,\left\{\lambda_{n}\right\} \subset(0,2 \alpha]$ and $\left\{\alpha_{n}\right\},\left\{\beta_{n}\right\},\left\{\gamma_{n}\right\},\left\{\delta_{n}\right\} \subset[0,1]$ such that

(i) $\beta_{n}+\gamma_{n}+\delta_{n}=1$ for all $n \geq 0$;

(ii) $\lim _{n \rightarrow \infty} \alpha_{n}=0$ and $\sum_{n=0}^{\infty} \alpha_{n}=\infty$;

(iii) $0<\liminf _{n \rightarrow \infty} \beta_{n} \leq \limsup _{n \rightarrow \infty} \beta_{n}<1$ and $\liminf _{n \rightarrow \infty} \delta_{n}>0$;

(iv) $\lim _{n \rightarrow \infty}\left(\gamma_{n+1} /\left(1-\beta_{n+1}\right)-\gamma_{n} /\left(1-\beta_{n}\right)\right)=0$;

(v) $0<\liminf _{n \rightarrow \infty} \lambda_{n} \leq \limsup _{n \rightarrow \infty} \lambda_{n}<2 \alpha$ and $\lim _{n \rightarrow \infty}\left|\lambda_{n+1}-\lambda_{n}\right|=0$.

Then the sequence $\left\{x_{n}\right\}$ converges strongly to $\bar{x}=P_{\mathrm{Fix}(S) \cap \Gamma \cap V I(A, C)} u$ and $(\bar{x}, \bar{y})$ is a solution of the general system (1.3) of variational inequalities, where $\bar{y}=P_{C}\left(\bar{x}-\mu_{2} B_{2} \bar{x}\right)$.

\section{Acknowledgments}

This research was partially supported by the National Science Foundation of China (10771141), Ph.D. Program Foundation of Ministry of Education of China (20070270004), Science and Technology Commission of Shanghai Municipality grant (075105118), and Shanghai Leading Academic Discipline Project (S30405). This research was partially supported by the Grant NSC 99-2115-M-110-004-MY3.

\section{References}

[1] F. E. Browder and W. V. Petryshyn, "Construction of fixed points of nonlinear mappings in Hilbert space," Journal of Mathematical Analysis and Applications, vol. 20, pp. 197-228, 1967.

[2] F. Liu and M. Z. Nashed, "Regularization of nonlinear ill-posed variational inequalities and convergence rates," Set-Valued Analysis, vol. 6, no. 4, pp. 313-344, 1998.

[3] J. C. Yao, "Variational inequalities with generalized monotone operators," Mathematics of Operations Research, vol. 19, no. 3, pp. 691-705, 1994.

[4] L.-C. Zeng, S. Schaible, and J. C. Yao, "Iterative algorithm for generalized set-valued strongly nonlinear mixed variational-like inequalities," Journal of Optimization Theory and Applications, vol. 124, no. 3, pp. 725-738, 2005.

[5] L.-C. Ceng and J.-C. Yao, "An extragradient-like approximation method for variational inequality problems and fixed point problems," Applied Mathematics and Computation, vol. 190, no. 1, pp. 205$215,2007$.

[6] M. A. Noor, "Some developments in general variational inequalities," Applied Mathematics and Computation, vol. 152, no. 1, pp. 199-277, 2004.

[7] Y. Censor, A. N. Iusem, and S. A. Zenios, "An interior point method with Bregman functions for the variational inequality problem with paramonotone operators," Mathematical Programming, vol. 81, no. 3, pp. 373-400, 1998.

[8] L.-C. Ceng, P. Cubiotti, and J. C. Yao, "An implicit iterative scheme for monotone variational inequalities and fixed point problems," Nonlinear Analysis: Theory, Methods E Applications, vol. 69, no. 8, pp. 2445-2457, 2008 
[9] H. K. Xu and T. H. Kim, "Convergence of hybrid steepest-descent methods for variational inequalities," Journal of Optimization Theory and Applications, vol. 119, no. 1, pp. 185-201, 2003.

[10] N. Nadezhkina and W. Takahashi, "Strong convergence theorem by a hybrid method for nonexpansive mappings and Lipschitz-continuous monotone mappings," SIAM Journal on Optimization, vol. 16, no. 4 , pp. 1230-1241, 2006.

[11] L.-C. Zeng, "Iterative algorithms for finding approximate solutions for general strongly nonlinear variational inequalities," Journal of Mathematical Analysis and Applications, vol. 187, no. 2, pp. 352-360, 1994.

[12] L.-C. Zeng, "Iterative algorithm for finding approximate solutions to completely generalized strongly nonlinear quasivariational inequalities," Journal of Mathematical Analysis and Applications, vol. 201, no. 1, pp. 180-194, 1996.

[13] L.-C. Ceng, S. Huang, and A. Petrussel, "Weak convergence theorem by a modified extragradient method for nonexpansive mappings and monotone mappings," Taiwanese Journal of Mathematics, vol. 13, no. 1, pp. 225-238, 2009.

[14] L.-C. Zeng and J.-C. Yao, "Mixed projection methods for systems of variational inequalities," Journal of Global Optimization, vol. 41, no. 3, pp. 465-478, 2008.

[15] L.-C. Zeng, L. J. Lin, and J. C. Yao, "Auxiliary problem method for mixed variational-like inequalities," Taiwanese Journal of Mathematics, vol. 10, no. 2, pp. 515-529, 2006.

[16] L.-C. Zeng, Q. H. Ansari, and S. Y. Wu, "Strong convergence theorems of relaxed hybrid steepestdescent methods for variational inequalities," Taiwanese Journal of Mathematics, vol. 10, no. 1, pp. 13$29,2006$.

[17] L.-C. Zeng, S.-M. Guu, and J.-C. Yao, "Iterative algorithm for completely generalized set-valued strongly nonlinear mixed variational-like inequalities," Computers \& Mathematics with Applications, vol. 50, no. 5-6, pp. 935-945, 2005.

[18] L.-C. Ceng and S. Huang, "Modified extragradient methods for strict pseudo-contractions and monotone mappings," Taiwanese Journal of Mathematics, vol. 13, no. 4, pp. 1197-1211, 2009.

[19] L.-C. Zeng, N. C. Wong, and J. C. Yao, "Convergence of hybrid steepest-descent methods for generalized variational inequalities," Acta Mathematica Sinica, vol. 22, no. 1, pp. 1-12, 2006.

[20] L.-C. Zeng, N. C. Wong, and J. C. Yao, "Convergence analysis of modified hybrid steepest-descent methods with variable parameters for variational inequalities," Journal of Optimization Theory and Applications, vol. 132, no. 1, pp. 51-69, 2007.

[21] L.-C. Ceng and J. C. Yao, "On generalized variational-like inequalities with generalized monotone multivalued mappings," Applied Mathematics Letters, vol. 22, no. 3, pp. 428-434, 2009.

[22] W. Takahashi and M. Toyoda, "Weak convergence theorems for nonexpansive mappings and monotone mappings," Journal of Optimization Theory and Applications, vol. 118, no. 2, pp. 417-428, 2003.

[23] N. Nadezhkina and W. Takahashi, "Weak convergence theorem by an extragradient method for nonexpansive mappings and monotone mappings," Journal of Optimization Theory and Applications, vol. 128, no. 1, pp. 191-201, 2006.

[24] L.-C. Zeng and J.-C. Yao, "Strong convergence theorem by an extragradient method for fixed point problems and variational inequality problems," Taiwanese Journal of Mathematics, vol. 10, no. 5, pp. 1293-1303, 2006.

[25] G. M. Korpelevič, "An extragradient method for finding saddle points and for other problems," Èkonomika i Matematicheskie Metody, vol. 12, no. 4, pp. 747-756, 1976.

[26] Y. Yao and J.-C. Yao, “On modified iterative method for nonexpansive mappings and monotone mappings," Applied Mathematics and Computation, vol. 186, no. 2, pp. 1551-1558, 2007.

[27] R. U. Verma, "On a new system of nonlinear variational inequalities and associated iterative algorithms," Mathematical Sciences Research Hot-Line, vol. 3, no. 8, pp. 65-68, 1999.

[28] R. U. Verma, "Iterative algorithms and a new system of nonlinear quasivariational inequalities," Advances in Nonlinear Variational Inequalities, vol. 4, no. 1, pp. 117-124, 2001.

[29] L.-C. Ceng, C. Wang, and J.-C. Yao, "Strong convergence theorems by a relaxed extragradient method for a general system of variational inequalities," Mathematical Methods of Operations Research, vol. 67, no. 3, pp. 375-390, 2008.

[30] Y. Yao, Y.-C. Liou, and S. M. Kang, "Approach to common elements of variational inequality problems and fixed point problems via a relaxed extragradient method," Computers $\mathcal{E}$ Mathematics with Applications, vol. 59, no. 11, pp. 3472-3480, 2010.

[31] G. L. Acedo and H.-K. Xu, "Iterative methods for strict pseudo-contractions in Hilbert spaces," Nonlinear Analysis: Theory, Methods E Applications, vol. 67, no. 7, pp. 2258-2271, 2007. 
[32] L.-C. Zeng, N.-C. Wong, and J.-C. Yao, "Strong convergence theorems for strictly pseudocontractive mappings of Browder-Petryshyn type," Taiwanese Journal of Mathematics, vol. 10, no. 4, pp. 837-849, 2006.

[33] T. Suzuki, "Strong convergence of Krasnoselskii and Mann's type sequences for one-parameter nonexpansive semigroups without Bochner integrals," Journal of Mathematical Analysis and Applications, vol. 305, no. 1, pp. 227-239, 2005.

[34] G. Marino and H.-K. Xu, "Weak and strong convergence theorems for strict pseudo-contractions in Hilbert spaces," Journal of Mathematical Analysis and Applications, vol. 329, no. 1, pp. 336-346, 2007.

[35] R. T. Rockafellar, "On the maximality of sums of nonlinear monotone operators," Transactions of the American Mathematical Society, vol. 149, pp. 75-88, 1970. 